\title{
Associations Between Implicit Motives and Salivary Steroids, 2D:4D Digit Ratio, Mental Rotation Performance, and Verbal Fluency
}

\author{
Oliver C. Schultheiss • Melanie Zimni
}

Received: 10 August 2014 / Revised: 1 October 2014 / Accepted: 3 October 2014 /

Published online: 15 October 2014

(C) Springer International Publishing 2014

\begin{abstract}
Both implicit motives and cognitive abilities have been shown to be associated with gonadal steroid hormones in previous research. Here, we present and test in a sample of 81 participants a conceptual model that links organizational and activational effects of gonadal steroids to implicit needs for power and affiliation, assessed with a picture-story method, 2D:4D digit ratio as a marker of organizational hormone effects, salivary measures of testosterone, progesterone, and estradiol as indicators of current (i.e., activational) effects, and spatial cognition and verbal ability, assessed with 3D mental rotation and verbal fluency tasks. Need for power showed both linear and curvilinear relationships with mental rotation performance and was associated with a more male digit ratio in individuals high in activity inhibition (AI), a picture-storybased measure of greater right-hemispheric engagement. Needs for affiliation and power interacted with $\mathrm{AI}$ and gender in predicting verbal fluency, with low-AI affiliation-motivated women and high-AI power-motivated women achieving higher verbal fluency scores than other participants. The opposite pattern was observed in men. Neither implicit motives nor digit ratio or cognitive ability measures were significantly associated with salivary hormones.
\end{abstract}

Keywords Implicit motives · Power motive - Affiliation motive - Activity inhibition Lateralization $\cdot$ Salivary hormones $\cdot$ Digit ratio $\cdot$ Cognitive ability $\cdot$ Mental rotation $\cdot$ Spatial cognition $\cdot$ Verbal fluency $\cdot$ Organizational vs activational hormone effects $\cdot$ Sex differences $\cdot$ Picture-story exercise $\cdot$ Content-coding

\section{Introduction}

There is evidence that implicit motives - that is, nonconsciously operating affect-based preferences for specific types of incentives (McClelland 1987; Schultheiss 2008) - are

O. C. Schultheiss $(\triangle) \cdot$ M. Zimni

Department of Psychology, Friedrich-Alexander University, Nägelsbachstrasse 49b, 91052 Erlangen, Germany

e-mail: Oliver.Schultheiss@fau.de 
closely linked to endocrine systems in humans (Schultheiss 2007, 2013; Stanton and Schultheiss 2009). The implicit need for power (n Power), which represents a capacity to experience one's dominant impact on others as pleasurable (Winter 1973), is associated with higher baseline levels of testosterone (T; Schultheiss 2013) and the release of adrenaline and noradrenaline in response to dominance challenges (McClelland 1989), the release of cortisol in response to defeat (Wirth et al. 2006), and, in response to successful dominance, the release of $\mathrm{T}$ in men (Schultheiss and Rohde 2002; Schultheiss et al. 2005) and estradiol (E) in women (Stanton and Schultheiss 2007; see also Stanton and Edelstein 2009). The implicit need for affiliation ( $\mathrm{n}$ Affiliation), a capacity to experience friendly, harmonious contact with others as pleasurable (Koestner and McClelland 1992), is associated with the release of progesterone $(\mathrm{P})$ in response to positive affiliative arousal (Brown et al. 2009; Schultheiss et al. 2004) and to affiliative stress (Wirth and Schultheiss 2006).

There is also evidence that gonadal steroid hormones play a role in fundamental cognitive processes, such as verbal ability and spatial cognition (e.g., Hampson 2002), as well as in the development of subtle morphological features beyond the fundamental female-male divide, such as the ratio between the 2 nd and the 4th digit (e.g., Lutchmaya et al. 2004). In the present research, we try to integrate these two lines of research by exploring for the first time whether implicit motives are associated with cognitive abilities that are susceptible to hormonal effects and whether steroid hormones such as $\mathrm{T}, \mathrm{E}$, and $\mathrm{P}$ are involved.

1. A conceptual framework of the relationship between implicit motives, hormones, and cognition

Our research was based on a conceptual framework that integrates known associations between hormones, cognitive abilities, and implicit motives and identifies gaps in the existing literature (see Fig. 1). A key feature of the model is the distinction between organizational and activational effects of gonadal steroid hormones on brain and body (Phoenix et al. 1959). Organizational effects are those that gonadal steroids like T and E exert on the developing brain and other tissues prenatally, permanently altering their structure and function. In contrast, activational effects of hormones are those through which variations in gonadal steroids later in life lead to non-permanent variations in the functions of neural and other tissues previously shaped by organizational effects. Both organizational and activational effects of gonadal steroids are widely documented for body, brain, and behavior (see, for instance, the textbooks by Becker et al. 2002; Nelson 2005).

On the backdrop of this fundamental distinction of endocrine function, we will first briefly review what is known about the relationship between hormones, motives, and cognitive abilities and then derive our predictions for the present research. In so doing, we focus on spatial skill as reflected in 3D mental rotation performance and verbal skill as reflected in verbal fluency. These cognitive abilities are particularly relevant because they tend to be sexually dimorphic in opposite directions (cf. Becker et al. 2005), with men typically outperforming women on mental rotation tasks and women outperforming men on verbal fluency tasks (Hampson 2002), and adaptive, because they are assumed to support hunting and within-gender competition (in the case of 


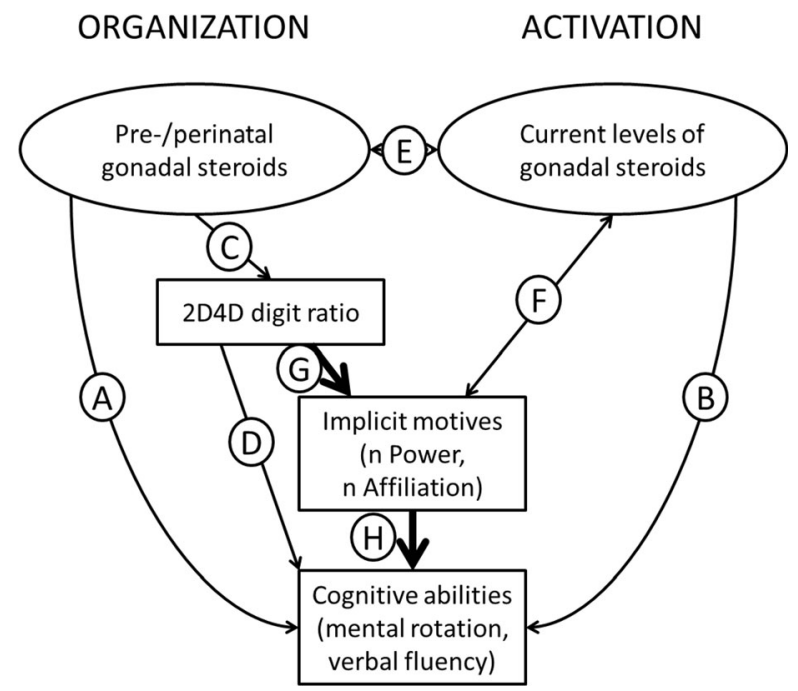

Fig. 1 Conceptual model linking organizational and activational effects of gonadal steroid hormones to cognitive abilities and implicit motives. Bold arrows represent pathways tested for the first time in the present research (i.e., Rumsfeldian known unknowns). Regular arrows represent previously studied relationships (i.e., Rumsfeldian known knowns). See text for further details on specific pathways

spatial cognition; see Ecuyer-Dab and Robert 2004) and group coordination and cohesion (in the case of verbal abilities; see Smith 2010). Because sexual differentiation of brain and body is largely under hormonal control during gestation (Breedlove and Hampson 2002), sex differences in spatial and verbal skills are assumed to be due to organizational effects of hormones, too, and therefore relevant for the study of endocrine effects on motivation and cognition (see Hampson 2002). As we will discuss in the following, this does not preclude activational effects of hormones. We structure our review based on the paths outlined in Fig. 1.

Many studies have explored the direct associations between cognition and prenatal steroids - corresponding to organizational effects (path A) - and steroid levels later in life, usually assessed concurrently with measures of cognition - corresponding to activational effects (path B). Some studies were able to investigate prenatal steroid effects on cognition directly through assessment of amniotic fluid steroid levels during gestation and longitudinal follow-up of cognitive development. Grimshaw et al. (1995) documented a curvilinear relationship between prenatal $\mathrm{T}$ and mental rotation ability in 7 -year old boys and girls. The curvature was the result of sex differences in the range of prenatal T: whereas the low $\mathrm{T}$ levels of girls predicted elevated mental rotation scores, the high $\mathrm{T}$ levels of boys were associated with reduced mental rotation scores (for related findings, see the meta-analysis on congenital adrenal hyperplasia [CAH] and mental rotation ability by Puts et al. 2008). While this study had a correlational design, a study by Roof and Havens (1992) in which T was administered to gonadally intact neonate rat pups suggests that the effect of perinatal $\mathrm{T}$ on spatial cognition is a causal one. As adults, female rats treated perinatally with $\mathrm{T}$, compared to controls, showed improved performance on a spatial navigation test, whereas T-treated males, which had already been exposed to high levels of $\mathrm{T}$ in utero, showed the opposite effect. $\mathrm{T}$ treatment was associated with an 
asymmetrical development of the hippocampus, a key structure for spatial navigation: the right hippocampus was larger in T-treated than control females. This finding is consistent with Geschwind and Galaburda's (1985) seminal theory of cerebral lateralization, which posits that higher perinatal $\mathrm{T}$ is associated with relatively better development of the right hemisphere (preferentially associated with spatial abilities) than the left hemisphere (preferentially associated with verbal abilities) up to a tipping point when $\mathrm{T}$ levels are so high that right-hemispheric development is compromised.

Effects of prenatal steroids on cognitive abilities (i.e., path A in Fig. 1) were also measured indirectly in a number of studies using the ratio of the second to fourth digit (2D:4D) as an indicator of prenatal hormone levels, with a lower ratio indicating the presence of greater prenatal androgen levels (path C). Evidence for such a marker function of 2D:4D for prenatal steroids comes from human studies that document a link in both genders between the ratio of $\mathrm{T}$ to $\mathrm{E}$ in amniotic fluid to postnatally assessed digit length (Lutchmaya et al. 2004), studies that document more male-typical 2D:4D in women with congenital adrenal hyperplasia, a dysfunction of the adrenals that is associated with heightened androgen release (Brown et al. 2002), and studies that use animal experiments to document a causal effect of prenatal $\mathrm{T}$ on the development of a male 2D:4D pattern (e.g., Talarovicova et al. 2009) and prenatal E on the development of a female 2D:4D pattern (e.g., Saino et al. 2007). 2D:4D digit ratio appears to be stable throughout postnatal development (e.g., Trivers et al. 2006). However, according to a meta-analysis by Puts et al. (2008), even though 2D:4D may represent a marker of prenatal steroid levels, it is not consistently related to mental rotation abilities (path D). To date, no similar meta-analysis exists for the relationship between 2D:4D and verbal ability (path D). But some individual studies report that a more female-typical digit ratio (reflecting lower prenatal androgen levels) is associated with better verbal fluency (Luxen and Buunk 2005), although this effect may be restricted to men and reversed in women, with a more male digit ratio predicting better fluency (Burton et al. 2005).

Regarding activational effects of current steroid levels on cognitive abilities (path B), some studies report that up to a point in the low male $\mathrm{T}$ range, higher levels of $\mathrm{T}$ are associated with better spatial abilities, including mental rotation, whereas levels in the high male range are associated with impaired spatial abilities (e.g., Gouchie and Kimura 1991; Moffat and Hampson 1996). However, when Puts et al. (2010) conducted the largest study on circulating T and mental rotation so far, with 337 participants, they did not find linear or curvilinear relationships between measures of both (for a similar null finding for activational effects of $\mathrm{T}$ on mental rotation, see Falter et al. 2006). Unlike Puts et al. (2010), who failed to examine menstrual cycle effects in their female participants, Hausmann et al. (2000) report that normally cycling women show better mental rotation performance in the follicular phase, characterized by low $\mathrm{P}$ and $\mathrm{E}$, than in the midluteal phase, characterized by high $\mathrm{P}$ and $\mathrm{E}$. This menstrual-cycle effect on mental rotation performance was recently replicated by Hampson et al. (2014), who concluded from salivary hormone assays that it is specifically high $\mathrm{E}$, but not $\mathrm{P}$, that accounts for impaired mental rotation. Some limited evidence also points to activational effects of gonadal steroids in verbal ability. Wolf and Kirschbaum (2002) observed a negative relationship between T and verbal fluency in men and Schattmann and Sherwin (2007) documented a positive effect of pharmacological reduction of T on verbal fluency in women. Regarding 
the relationship between E and verbal ability, Hampson (1990a) observed an increase of verbal fluency from the follicular to the midluteal phase. Effects suggestive of a positive association between $\mathrm{E}$ and use of signs have also been observed in a primate trained in sign language (Patterson et al. 1991). However, the link between $\mathrm{E}$ and verbal fluency appears to be difficult to replicate, sometimes even within the same laboratory (see Hampson 1990b).

We conclude from this review that there are profound organizational effects of preor perinatal steroids (particularly $\mathrm{T}$ and $\mathrm{E}$ ) on mental rotation and also on digit ratio, but that there is no systematic association between digit ratio as a marker of such organizational effects and mental rotation performance. A more female digit ratio appears to be related to better verbal fluency, particularly in men. Some evidence exists for activational effects of $\mathrm{T}$ and $\mathrm{E}$ on mental rotation, but the evidence for $\mathrm{T}$ in particular is inconsistent across studies. Some limited evidence points to a negative association of verbal fluency with current $\mathrm{T}$, and a positive association with current $\mathrm{E}$ levels.

Research has also addressed the issue of whether prenatal gonadal steroids are associated with gonadal steroids later in life (path E), an issue that would limit the interpretation of prenatal steroids as having mainly organizational effects. In a synthesis of two new empirical studies and a meta-analysis, Hönekopp et al. (2007) found no evidence that 2D:4D as a marker of the prenatal endocrine milieu is associated with $\mathrm{T}$, $\mathrm{E}$, or $\mathrm{P}$ in adulthood.

\section{The present study}

We summarized in the introductory paragraph research that links implicit motives to current levels of gonadal steroids (path F), with n Power predicting situation-dependent changes in $\mathrm{E}$ and $\mathrm{T}$ and $\mathrm{n}$ Affiliation being associated with $\mathrm{P}$. In the present research, we aimed to examine two hitherto untested paths, namely the relationship between implicit motives and prenatal steroids, approximated by a measure of digit ratio (path G), and implicit motives and sexually dimorphic cognitive abilities (path $\mathrm{H}$ ). The former path addresses the question whether implicit motivational needs for power and affiliation are shaped prenatally by the intrauterine endocrine milieu. To the extent that this path exists, one should be able to observe continuity in the behavioral markers of power and affiliation motivation from early childhood into adulthood. For $n$ Power, such evidence comes from a longitudinal study in which more aggressive-assertive behavior in boys and girls aged up to 5 years could be linked to higher levels of $n$ Power, assessed in the same individuals at age 32 (McClelland and Pilon 1983). In other words, $n$ Power has its roots in early-childhood behaviors that have been linked to organizational (Liu et al. 2012; Pasterski et al. 2007) and activational effects of $\mathrm{T}$ on aggression and dominance (Mazur and Booth 1998). Less is known about childhood behavioral correlates of adult $\mathrm{n}$ Affiliation. The study by McClelland and Pilon (1983) points to a role of parental responsiveness to the child's crying and the use of praise in the development of this motive - that is, socialization factors -, but did not identify specific childhood behaviors that predict later $\mathrm{n}$ Affiliation levels. Moreover, $\mathrm{n}$ Affiliation has been linked to $\mathrm{P}$, which so far has not been shown to play a role in organizational effects reflected in digit ratio differences, but not to $\mathrm{T}$ or $\mathrm{E}$. We therefore expected $n$ Power to be associated with a more male pattern of digit ratio, reflecting greater prenatal androgen exposure, but did not articulate a specific hypothesis 
regarding the association between $\mathrm{n}$ Affiliation and digit ratio. Our analyses will be exploratory in this regard.

Path $\mathrm{H}$ from implicit motives and sexually dimorphic cognitive abilities addresses the question to what extent implicit motives themselves may mediate organizational and/or activational effects of steroids on cognition. Of the two motives examined in the present research, only $\mathrm{n}$ Affiliation shows a gender difference, with women typically scoring higher than men (Pang and Schultheiss 2005; Schultheiss and Brunstein 2001). Schultheiss et al. (2003) suggested that this gender difference may be due to activational effects of $\mathrm{P}$, because $\mathrm{n}$ Affiliation was higher in women using progestin-based oral contraceptives than in normally cycling women and increases in salivary $\mathrm{P}$ preceded increases in n Affiliation in the latter women. In the present research, we hypothesized that because $\mathrm{n}$ Power has been linked to $\mathrm{T}$ in both men and women (see Schultheiss 2013; Schultheiss et al. 2005), it would predict mental rotation ability. We expected n Affiliation to predict verbal fluency for the following reasons. First, the gender difference observed for this motive is in the same direction as the gender difference for verbal fluency (see Hampson 2002), Second, verbal communication is a critical means for establishing and maintaining close, friendly relationships; that is, for the pursuit of core affiliation incentives and concomitant increases in P (Brown et al. 2009). Third, past studies document that $\mathrm{n}$ Affiliation is associated with more oral and written communication (Lansing and Heyns 1959; McAdams and Constantian 1983), a finding that is consistent with the notion of enhanced verbal skills in affiliation-motivated individuals.

We tested these hypotheses in a cross-sectional study in which we measured $n$ Power and $\mathrm{n}$ Affiliation with a standard picture-story measure for the assessment of implicit motives (see Schultheiss and Pang 2007). This procedure also allowed us to measure participants' activity inhibition (AI), a linguistic marker of lateralization towards righthemispheric functions under stressful conditions (Schultheiss et al. 2009) that has been frequently found to moderate behavioral effects of $\mathrm{n}$ Power (e.g., McClelland and Boyatzis 1982; McClelland and Pilon 1983; Rösch et al. 2013), n Affiliation (Mason and Blankenship 1987), or the balance between $n$ Power and $n$ Affiliation, particularly in research on immune system function and health (Jemmott 1987; McClelland 1989). In light of Geschwind and Galaburda's (1985) theorizing about androgens leading to enhanced right-lateralized brain development, we considered this variable a potential moderator of the hypothesized findings. We measured digit ratio as a marker of prenatal steroid exposure, and thus organizational effects of $\mathrm{T}$ and $\mathrm{E}$, and current levels of salivary $\mathrm{T}, \mathrm{E}$, and $\mathrm{P}$ to test for activational effects. Cognitive abilities were assessed through an adaptation of Vandenberg and Kuse's (1978) 3D mental rotation task and, for verbal fluency, as a combination of adaptations of oral fluency (Thurstone and Thurstone 1962) and expressional fluency tasks (Christensen and Guilford 1958) used in previous research on hormones and verbal ability (e.g., Burton et al. 2005; Hampson 1990a).

\section{Method}

Participants

In 2010, 81 participants, who were contacted on the premises of Friedrich-Alexander University, Erlangen, Germany, were recruited for a "study on hormones, imagination, 
and cognition". Sample size represented a compromise between an intended ideal power of .80 for small to medium effect sizes $(r \sim .25)$ obtained in previous research on motives, hormones, and cognition (e.g., Schultheiss et al. 2003; Schultheiss and Hale 2007; Schultheiss et al. 2004) and the requirement for completing a diploma thesis at Friedrich-Alexander University within 6 months. The sample consisted of 37 men, 18 naturally cycling (NC) women, and 26 women taking oral contraceptives (OC). On average, participants were 23.13 years old $(\mathrm{SD}=3.38$, Min $=18$, Max $=38$ ). Eight participants self-identified as left-handed. No participant was enrolled in psychology.

\section{Design}

The study had a correlational design. For data-analytic purposes, however, implicit motive measures and participant gender (male, female) and group (men, NC women, $\mathrm{OC}$ women) were designated as predictor variables and measures of digit ratio, mental rotation performance, and verbal fluency were designated as dependent variables. Hormone measures were used variably as predictor and dependent variables.

\section{Procedure}

To minimize variations in hormonal measures, all testing took place in the afternoon, after $3 \mathrm{pm}$. All tasks and questionnaires were presented on Windows XP computers with 24" LCD monitors, using the software Inquisit 3.0 by Millisecond Software (Seattle, WA). Participants first completed a picture-story measure of implicit motives and AI and then worked on a demographic questionnaire while providing one saliva sample. Next, they completed a mental rotation task and a verbal fluency task. After participants had completed all tasks (including a self-report measure of motives, an emotion recognition task, and a lateralized attention task that were not relevant for the questions and hypotheses of this article), the experimenter used a photocopier to take pictures of participants' hands for later determination of digit ratio. Participants were debriefed, thanked, and paid $€ 10$ for their participation.

\section{Motive Measures}

Participants worked on the 6-picture PSE described by Pang and Schultheiss (2005), following standard instructions for computer administration provided in Schultheiss and Pang (2007). Stories were later coded for motive imagery by two trained scorers using Winter's (1994) Manual for Scoring Motive Imagery in Running Text. According to the manual, $\mathrm{n}$ Power is coded whenever a story character acts in a strong, forceful manner; controls or manipulates others; tries to persuade or convince others; provides unsolicited help or advice; wants to impress others, has a concern with fame, prestige; or elicits strong, non-reciprocal emotions in others. $n$ Affiliation is coded when someone experiences positive affect in the context of a relationship; experiences negative affect about the disruption of a relationship and wants to mend it; engages in companionate activity with others; or provides nurturant support to others. (Stories were also scored for achievement imagery; however, these scores have no bearing on the hypotheses and results reported here.) Scorers had previously exceeded $85 \%$ interscorer agreement on calibration materials prescored by an expert that are contained in 
the manual. Interrater reliability (Pearson correlations of each participant's raw motive scores) was good, with .82 for $\mathrm{n}$ Power and .86 for $\mathrm{n}$ Affiliation. Word count and AI (i.e., the frequency of the German negation nicht) were determined through a MATLAB utility described in Schultheiss et al. (2008). PSE protocol length ( $M=580$ words, $S D=154$ words) was significantly correlated with participants' overall motive scores, averaged across both coders, for n Power $(M=4.99, S D=2.46)$, $r=.65$, and $\mathrm{n}$ Affiliation $(M=5.80, S D=2.41), r=.36$, as well as with their AI scores $(M=5.60, S D=3.55), r=.49$, all $p \mathrm{~s}<.001$. Following Schultheiss and Pang's (2007) recommendation, we corrected motive and AI scores by regression for word count and converted the residuals to $\mathrm{z}$ scores. We used these standardized scores in subsequent data analyses.

\section{Salivary Hormones}

Saliva samples were collected and processed according to the recommendations given by Schultheiss and Stanton (2009). All hormone assays were conducted using solidphase Coat-A-Count ${ }^{125}$ I radioimmunoassays for T (TKTT), E (TKE2), and P (TKPG) provided by Siemens Healthcare Diagnostics GmbH (Eschborn, Germany), following validated protocols for the assessment of salivary T, E, and P (Liening et al. 2010; Schultheiss et al. 2003; Stanton and Schultheiss 2007; a 24-h preincubation at room temperature was added for P). All participant samples were assessed in duplicate and estimates were averaged for each sample for later analyses. For T, intraassay CV was $10 \%$, and analytical sensitivity (B0 - 3 SD) was $5 \mathrm{pg} / \mathrm{mL}$. For diluted control samples, analytical recovery was $157 \%$ for low $(10 \mathrm{pg} / \mathrm{mL}), 80 \%$ for medium $(90 \mathrm{pg} / \mathrm{mL})$, and $78 \%$ for high concentrations $(152 \mathrm{pg} / \mathrm{mL})$. For E, intraassay CV was $17 \%$, and analytical sensitivity was $0.02 \mathrm{pg} / \mathrm{mL}$. For diluted control samples, analytical recovery was $104 \%$ for low $(2.07 \mathrm{pg} / \mathrm{mL})$ and $76 \%$ for high concentrations $(3.04 \mathrm{pg} / \mathrm{mL})$. For P, interassay $\mathrm{CV}$ (determined for a male and a female saliva pool across two assays) was $1 \%$, intraassay CV was $12 \%$, and analytical sensitivity was $1 \mathrm{pg} / \mathrm{mL}$. For diluted control samples, analytical recovery was $105 \%$ for low $(21 \mathrm{pg} / \mathrm{mL})$ and $111 \%$ for high concentrations $(111 \mathrm{pg} / \mathrm{mL})$. Previous research shows that a single measurement of gonadal steroids in saliva yields a reliable estimate of a person's typical hormone level (e.g., Sellers et al. 2007; Stanton and Edelstein 2009). Because salivary P was not normally distributed due to NC women with high luteal-phase levels, we ran all statistical tests on $\log$-transformed $(\log [1+\mathrm{P}])$ values, but report untransformed descriptive statistics for this measure.

\section{Digit Ratio}

Participants placed the surfaces of both hands side by side on the platen of a copier (Nashuatec MP 2000 Aficio) and the experimenter took a copy. Digit lengths (in mm) for the 2 nd and the 4 th digits were later determined with a ruler from these images by measuring the distance between the tip and the midpoint of the bottom crease of each finger. This method has been shown to provide measurements with satisfactory reliability (Kemper and Schwerdtfeger 2009). Digit ratio was calculated for each hand by dividing the length of the 2nd digit (left hand: $M=70.64 \mathrm{~mm}, S D=4.94 \mathrm{~mm}$; right hand: $M=69.68 \mathrm{~mm}, S D=4.78 \mathrm{~mm}$ ) by the length of the 4 th digit (left hand: $M$ 
$=72.65 \mathrm{~mm}, S D=5.23 \mathrm{~mm}$; right hand: $M=73.16 \mathrm{~mm}, S D=4.98 \mathrm{~mm}$ ). Because we decided to include a measure of digit ratio in this study after data collection had already begun, we obtained valid measurements of digit ratio from 50 participants only (25 men, $11 \mathrm{NC}$ women, 14 OC women).

\section{Mental Rotation Task}

To assess participants' mental rotation performance, we adapted the paper-and-pencil task by Vandenberg and Kuse (1978) for administration on the PC. Target items were taken from the mental rotation stimulus library developed by Peters and Battista (2008). They consisted of $2 \times 12$ 3D drawings of abstract figures, each assembled from 10 cubes. Each target was presented in white on black background in the upper half of the screen. In the lower half of the screen, two horizontally or vertically rotated versions of the target item were shown along with two horizontally or vertically rotated versions of the mirror image of the target item. Thus, only the former two items could be rotated into congruence with the target item (=correct items), but not the latter two (=incorrect items). On each trial, correct and incorrect items were assigned randomly to the four positions in the lower half of the screen. Participants had to indicate which 2 items represented rotated versions of the target item. A correct response was scored as 1 only if both correct items were identified. All other responses were scored as incorrect (0).

Participants had three training trials to get acquainted with the task and test their understanding of the instructions. Next, participants worked on 2 blocks of 12 targets each, with a time limit of $3 \mathrm{~min}$ per block and a $30 \mathrm{~s}$ break between blocks. The sequence of target item presentations within each block was randomized before the study was run, but was the same for each participant. Within each block, no time limit for individual items was given; participants were instructed to correctly solve as many items as possible within the allotted time. Mental rotation task performance had satisfactory retest stability $(r=.64)$ from the first block $(M=4.86, S D=1.94)$ to the second block $(M=5.56, S D=1.98)$. We therefore created an overall mental rotation score by summing correct responses across both blocks.

\section{Verbal Fluency Task}

To assess participants' verbal fluency, we administered the tasks prefixes (Wortanfänge), suffixes (Wortenden), and sentences (Vierwort-Sätze) from a standardized verbal creativity test (Verbaler Kreativitäts Test, VKT; Schoppe 1975), which is based on earlier tests by Christensen and Guilford (1958) and Thurstone and Thurstone (1962). The prefix task required participants to generate and type into a window on the screen as many different words as possible starting with the common German prefixes ver-and $d e$ - (90 s per prefix). Similarly, the suffix task required participants to generate as many different words as possible ending with the common German suffixes -los and -ing (90 s per suffix). The sentence task required participants to generate as many meaningful sentences from 4 initial letters as possible within the allotted time $(150 \mathrm{~s}$ each for R-S-A-M and D-L-P-W; a correct English solution for the first item would be "Ricky makes a salad"). Six participants failed to complete the entire set of VKT tasks or individual tasks and their data were discarded from all further analyses concerning verbal fluency (hence, $n=75 ; 34$ men, $17 \mathrm{NC}$ women, $24 \mathrm{OC}$ women). 
Because item pairs for each VKT subtest were substantially correlated ( $r \mathrm{~s}>.38)$, we created sum scores for prefixes $(M=26.57, S D=8.09)$, suffixes $(M=12.83, S D=5.12)$, and sentences $(M=10.08, S D=3.55)$. Prefixes correlated at .69 with suffixes and at .59 with sentences; suffixes correlated at .60 with sentences (all $p \mathbf{s}<.0001$ ).

Because we were concerned that participants' performance scores might confound typing speed with verbal fluency, we additionally had them type the numbers 1 through 10 as words within $10 \mathrm{~s}$. The number of completed number words was not significantly correlated with VKT task scores $(p s>.10)$ and neither did it alter the results reported below when we included it as a covariate in regression analyses. Thus, results for VKT scores were not due to typing speed.

\section{Results}

Descriptive Statistics and Correlations

Table 1 provides an overview of descriptive statistics, both for the whole sample and broken down by group (men, $\mathrm{NC}$ women, $\mathrm{OC}$ women), as well as the correlations between all study variables. Group did not have an effect on participants' n Power and AI levels. However, replicating previous findings (Schultheiss et al. 2003), OC women had higher $\mathrm{n}$ Affiliation than men. NC women's n Affiliation was in between these groups and differed from each at the trend level $(p s<.10)$. Group also had the expected effect on salivary hormone levels, with men having about five- to seven-fold higher $\mathrm{T}$ than $\mathrm{NC}$ and $\mathrm{OC}$ women, and $\mathrm{OC}$ women having higher $\mathrm{E}$ and $\mathrm{P}$ than $\mathrm{NC}$ women. Men's E differed from both OC and NC women's. Group had no reliable effect on other variables, including mental rotation performance. However, when we combined NC and $\mathrm{OC}$ women, they showed the expected trend toward lower mental rotation scores $(M=9.77, S D=3.57)$ compared to men, $t(79)=1.81, p=.07$.

After controlling for the substantial effects of group on salivary hormone levels, all three hormones were positively and significantly associated with each other (see Table 1). There were no significant associations between $n$ Power and $n$ Affiliation with salivary hormones for the entire sample. Targeted analyses of the relationship between $\mathrm{n}$ Power and $\mathrm{T}$ in men $(r=-.22, n s)$ and between $\mathrm{n}$ Power and $\mathrm{E}$ in $\mathrm{NC}$ women $(r=.25, n s)$ were inconclusive, although the latter effect is consistent with the positive correlation $(r=.44$; for the difference, $z=-0.85, n s)$ between both variables observed in NC women by Stanton and Schultheiss (2007). Likewise, analyses of associations between $\mathrm{n}$ Affiliation and $\mathrm{P}$ across groups were inconclusive, with these variables being correlated at .02 in men, .08 in NC women, and .35 in OC women (all $n s$ ). As expected, digit ratio was not significantly related to salivary hormones with the exception of $\mathrm{P}$, for which we found a negative correlation. Contrary to expectations, none of the hormones was significantly associated with mental rotation performance or verbal fluency, either in the overall sample (Table 1) or differentially by group. We also tested for a curvilinear relationship between $\mathrm{T}$ and mental rotation scores, but without significant results.

As hypothesized, $n$ Power was negatively associated with 2D:4D ratio, whereas the reverse was true for mental rotation performance. Correlations of these variables with $n$ Affiliation were in the opposite direction. However, only the association between $n$ 


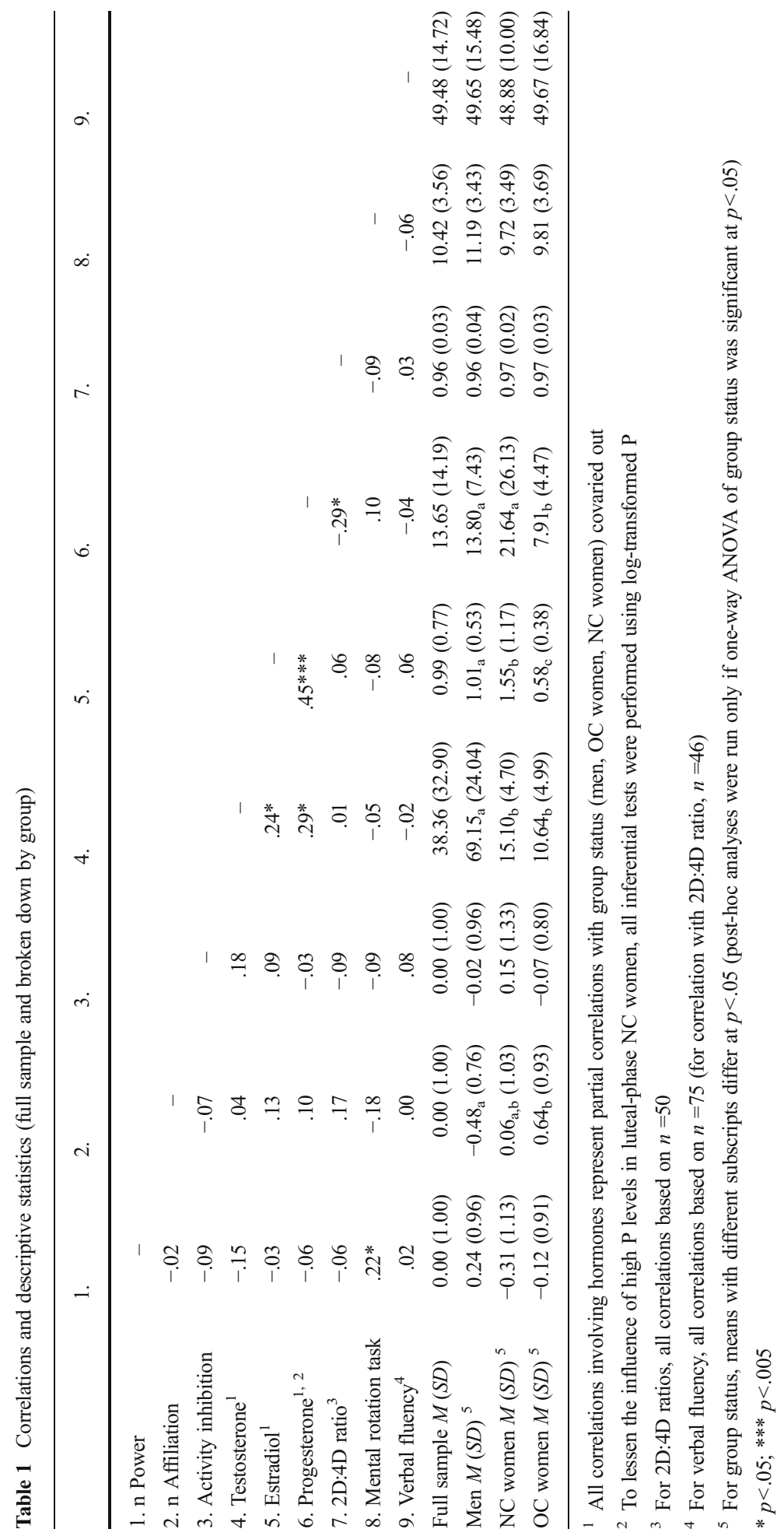


Power and mental rotation became significant. Neither $\mathrm{n}$ Power, nor $\mathrm{n}$ Affiliation was directly related to verbal fluency. However, more detailed analyses revealed that motive measures were associated substantially, but in a more complex fashion than reflected at the level of zero-order correlations, with digit ratio, mental rotation, and verbal fluency.

\section{Digit Ratio}

A repeated-measures analysis with hand as within-subjects factor and $\mathrm{n}$ Power and AI as well as their interaction term as between-subjects factors revealed a significant $n$ Power x AI effect, $F(1,46)=5.72, p=.02$ (for the full model, $R^{2}=.121$ ), that was not moderated by hand, $F(1,46)=1.37, p=.25$. When we added gender as another between-subjects factor, it failed to moderate the $\mathrm{n}$ Power $\mathrm{x}$ AI interaction, too, $F(1$, $42)=0.04, p=.84$. To follow up on the $\mathrm{n}$ Power $\mathrm{x}$ AI effect, we first split AI slightly below the median of those participants who had 2D:4D data available and repeated the analysis with the split variable instead of the quantitative AI variable. We were able to capture the original effect without much loss of test power with the dichotomous AI variable - for the $\mathrm{n}$ Power $\mathrm{x}$ split $\mathrm{AI}$ interaction, $F(1,46)=4.59, p=.04$. We therefore decided to run follow-up analyses and illustrate the effect by splitting the design using the dichotomous AI variable. As Fig. 2 shows, in individuals high in AI, $n$ Power tended to be associated with a more male $2 \mathrm{D}: 4 \mathrm{D}$ ratio, $B=-0.0109, S E=0.0065$, $r=-.31, t(26)=-1.67, p=.11$, whereas in individuals low in AI, it tended to be associated with a more female $2 \mathrm{D}: 4 \mathrm{D}$ ratio, $B=0.0103, S E=0.0073, r=.30, t(20)=1.40, p=.18$.

\section{Mental Rotation}

Further explorations into the positive association between $n$ Power and mental rotation performance described above revealed that it was moderated by gender, with men showing a non-significant negative association between $\mathrm{n}$ Power and mental rotation performance, $B=-0.134, S E=0.603, r=-.04, t(35)=-0.22, p=.83$, and women showing a significant positive association between the two variables, $B=1.302, S E=0.514$,

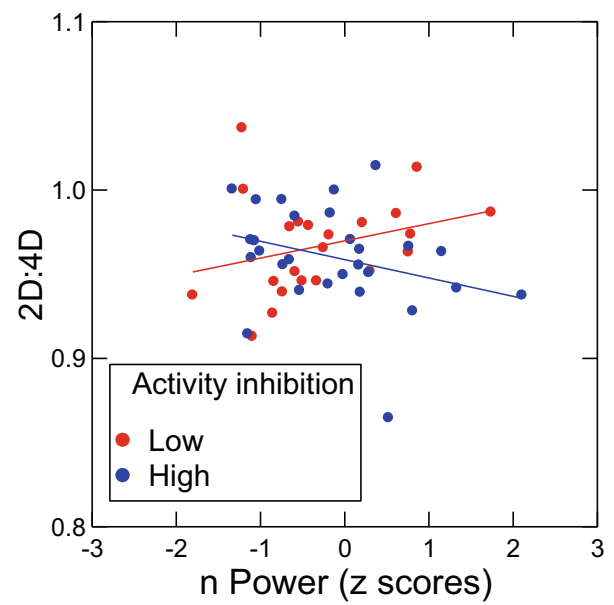

Fig. 2 Interaction effect of $n$ Power and activity inhibition on 2D:4D digit ratio (averaged across both hands) 
$r=.36, t(42)=2.54, p=.02$ (for the interaction, $B=1.436, S E=0.789, t(77)=1.82$, $p=.07)$. However, the linear association between $\mathrm{n}$ Power and mental rotation performance also turned out to be superseded by a significant quadratic effect of n Power, $B=$ $-0.657, S E=0.253, t(78)=-2.60, p=.01$ (for the full model including linear and quadratic effects, $\left.R^{2}=.126, F(2,78)=5.63, p=.005\right)$, and this effect was not moderated by gender. Figure 3 illustrates both the linear and the quadratic effects of $\mathrm{n}$ Power on mental rotation performance.

\section{Verbal Fluency}

A repeated-measures analysis with the three subtests of the VKT (prefixes, suffixes, sentences) as within-subjects factor and gender, $\mathrm{n}$ Power, $\mathrm{n}$ Affiliation, and AI as between-subjects factors yielded two three-way interactions: a Gender x $\mathrm{n}$ Power $\mathrm{x}$ AI effect, $F(1,63)=4.39, p=.04$, and a Gender x n Affiliation x AI effect, $F(1,63)$ $=5.92, p=.02$. After aggregating the three subtest scores into an overall verbal fluency score (for the full model, $R^{2}=.167$ ), inspection of the signs of these interaction terms suggested that they could be simplified algebraically into one three-way interaction of participant gender, AI, and a motive difference score, with higher (positive) scores indicating a predominance of $\mathrm{n}$ Affiliation over $\mathrm{n}$ Power (for this interaction term, $B=$ $-9.115, S E=2.861, t(67)=-3.19, p=.002$ ). For follow-up analyses and graphical illustrations of this effect, we split the sample by AI level in the manner described above. The Predominant Motive x Gender effect was significant, but in different directions, both for individuals low in AI, $B=14.567, S E=4.081, t(30)=-3.55$, $p=.001$, and for individuals high in AI, $B=-8.438, S E=3.159, t(37)=2.67, p=.01$. As illustrated in Fig. 4, effects of predominant $n$ Affiliation on verbal fluency depended critically on AI and gender. Among low-AI participants, a predominant $\mathrm{n}$ Affiliation was significantly associated with enhanced verbal fluency in women, $B=7.408, S E$ $=1.446, r=.80, t(15)=5.12, p=.0001$, but not in men, $B=-7.059, S E=4.163, r=-.40$, $t(15)=1.70, p=.11$. In contrast, among high-AI participants, a predominant $\mathrm{n}$ Affiliation was significantly associated with impaired verbal fluency in women, $B=-5.473$,

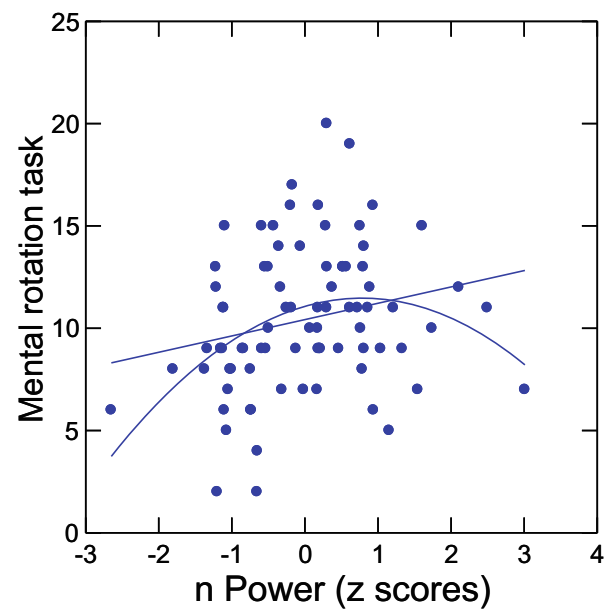

Fig. 3 Linear and quadratic associations between $n$ Power and mental rotation task performance 

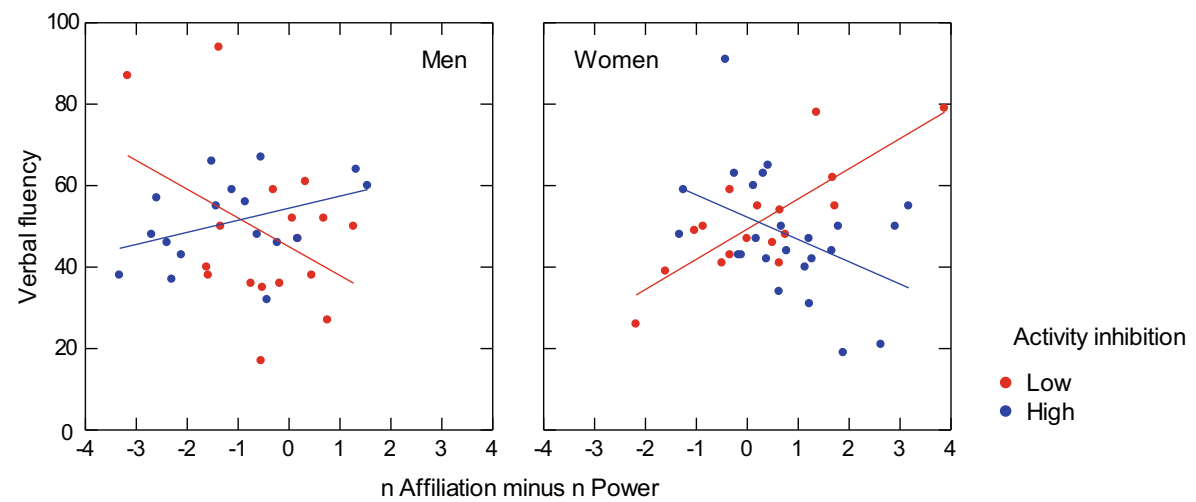

Fig. 4 Interaction effect of gender, motive (n Affiliation minus n Power), and activity inhibition on the composite measure of verbal fluency

$S E=2.471, r=-.43, t(22)=-2.21, p=.04$, but again not in men, $B=2.965, S E=1.800$, $r=.39, t(15)=1.65, p=.12$. Although the Predominant Motive slopes for verbal fluency were not significantly different from zero for low- and high-AI men, they did differ significantly from each other, as indicated by a Predominant Motive $\mathrm{x}$ AI interaction in the male subsample, $B=10.024, S E=4.289, t(30)=2.34, p=.03$.

\section{Discussion}

In this study, we tested whether there is evidence of an organizational effect of pre- or perinatal gonadal steroids on adult implicit motivational needs for power and affiliation and whether these motives in turn predict cognitive abilities that are sexually dimorphic due to organizational and/or activational hormone effects. In doing so, we drew on a conceptual framework that synthesized what is known about the relationships between gonadal steroids, implicit motives, and cognitive abilities (see Fig. 1).

In support of our hypothesis of an involvement of organizational hormonal effects on adult implicit motives, we found $\mathrm{n}$ Power to be associated with digit ratio, a marker of the intrauterine endocrine milieu, but in a manner that was moderated by participants' AI, a measure of latent brain lateralization. In individuals high in AI, characterized by a tendency towards right-hemispheric engagement during stress, $\mathrm{n}$ Power tended to be associated with a more male digit ratio pattern suggestive of a preponderance of high $\mathrm{T}$ and low $\mathrm{E}$ in early development. In individuals low in AI, in contrast, $\mathrm{n}$ Power tended to be associated with a more female digit ratio pattern suggestive of low $\mathrm{T}$ and/or high $\mathrm{E}$ in early development. $\mathrm{n}$ Affiliation was associated with a more female pattern of digit ratio, but this association failed to reach significance. Although the sample size for analyses involving 2D:4D digit ratio was smaller than for our other analyses, a recent replication of the $\mathrm{n}$ Power $\mathrm{x}$ AI effect on digit ratio in two other, much larger samples (Oxford et al. in preparation) suggests that our finding is not due to chance.

$\mathrm{n}$ Power was also associated with 3D mental rotation ability. While the relationship first emerged as a linear one, with higher $\mathrm{n}$ Power being associated with better mental rotation, intriguingly there was also a curvilinear association, with higher $\mathrm{n}$ Power 
being associated with better mental rotation only up to medium levels of $\mathrm{n}$ Power and with worse mental rotation for very high levels in $\mathrm{n}$ Power. In addition, we also observed that the positive linear relationship between $n$ Power and mental rotation tended to emerge more clearly for women than for men. At first blush, this finding is reminiscent of studies in which higher prenatal or current $\mathrm{T}$ was associated better mental rotation performance in women, but not in men, and this effect contributed to an curvilinear relationship between $\mathrm{T}$ and mental rotation performance overall (e.g., Grimshaw et al. 1995; Moffat and Hampson 1996). However, although n Power is functionally linked to T (Stanton and Schultheiss 2009), there is no strong gender difference for $\mathrm{n}$ Power like there is for $\mathrm{T}$, neither in this study nor in others (e.g., Pang and Schultheiss 2005; Schultheiss and Brunstein 2001). The $\mathrm{n}$ Power by gender interaction effect on mental rotation therefore cannot explain the curvilinear effect of $\mathrm{n}$ Power on mental rotation in the full sample. Overall, however, we suggest that the observed association between $\mathrm{n}$ Power and mental rotation performance on the one hand and the lack of direct evidence for activational effects of salivary $\mathrm{T}$ and $\mathrm{E}$ on mental rotation on the other is consistent with the notion that $\mathrm{n}$ Power is partly rooted in organizational effects of perinatal effects of $\mathrm{T}$ (and perhaps $\mathrm{E}$ ). This may also help explain why behaviors that can be linked to adult $n$ Power already emerge in early childhood (cf. McClelland and Pilon 1983).

Results for verbal fluency turned out to be more complex than expected. First of all, unlike mental rotation performance, for which we did find the expected gender difference at the trend level, women and men did not significantly differ in verbal fluency, and neither did we find evidence for an activational effect of $E$ on this variable. These findings contrast with earlier reports describing verbal fluency as a sexually dimorphic ability (Burton et al. 2005; Hampson 2002) and linking it to variations in $\mathrm{E}$ (e.g., Hampson 1990a). However, as we have already pointed out, these associations appear difficult to replicate (cf. Hampson 1990b; Miller and Halpern 2014).

Second, we also failed to observe the hypothesized direct association between $n$ Affiliation and verbal fluency. As it turned out, $\mathrm{n}$ Affiliation did predict verbal fluency once it was directly contrasted with participants' $n$ Power scores, and gender and AI were taken into consideration. Women's verbal fluency was enhanced to the degree that they were predominantly affiliation-motivated (i.e., their $n$ Affiliation scores were considerably higher than their $\mathrm{n}$ Power scores) and had low AI scores, which according to previous research are a marker of greater left-hemispheric dominance (cf. Schultheiss et al. 2009). The relationship was the opposite for women high in AI (i.e., greater righthemispheric dominance), who turned out to be more verbally fluent the more their $n$ Power exceeded their $n$ Affiliation levels. A weaker AI x Predominant Motive effect was present in the male subsample. Here, a predominant $n$ Affiliation was associated with better verbal fluency among high-AI participants and with worse verbal fluency among low-AI participants. These findings suggest that verbal fluency may not be sexually dimorphic per se, but is associated differently with motivational needs in men and women, with high verbal fluency being the result of either an inhibited power motive or an uninhibited affiliation motive in women, and of an uninhibited power motive or an inhibited affiliation motive in men. If that is the case, it is not surprising that previous studies only obtained rather inconsistent results with regard to gender differences in verbal fluency or its association with markers of organizational and activational hormone effects (Miller and Halpern 2014). Our findings suggest that 
effects of gender and gonadal steroids on verbal fluency are not simple and direct, but actually have rather complex motivational roots and may therefore be difficult to detect. We therefore think that it may be worthwhile to examine the relationship between verbal fluency and gender-dependent effects of motives and hormones in more detail in future studies.

We were unable to find any statistically reliable associations between current levels of salivary steroids and implicit motive measures. As Schultheiss (2013) has pointed out, substantial associations between circulating hormones and motivational needs should not be expected because implicit motives will be associated with steroid hormones only in the presence of suitable incentive cues (which we did not introduce or vary in the present study) and hormone levels are themselves influenced by many other factors besides motivation. The only exception from this general rule, although an indirect one, was the association between gender and OC use on the one hand and $n$ Affiliation on the other. Replicating an earlier observation by Schultheiss et al. (2003), we found that women using OCs, which typically contain progestins or a mix of estrogens and progestins, had higher levels of $\mathrm{n}$ Affiliation than women not on OCs or men. Due to the correlational nature of our data, however, we cannot say for sure whether OC use increased women's n Affiliation, or women high in n Affiliation were more inclined to use OCs, or another variable played a causal role in both OC use and $n$ Affiliation.

\section{Limitations and Future Directions}

Although the present findings are broadly consistent with our conceptual framework linking organizational and activational effects of hormones to implicit motives and cognitive abilities, we acknowledge that support for the model must come from further studies that incrementally overcome the limitations of our cross-sectional design and its inherent ambiguities with regard to cause, consequence, and potential third-variable effects. One key issue is the role of organizational effects on implicit motives. In the present study, we did not measure pre- or perinatal $\mathrm{T}$ and $\mathrm{E}$ directly, but through a marker (i.e., digit ratio). As we have already pointed out, it is unlikely that variance in this marker is exclusively due to hormonal influences, and it may therefore represent only a noisy messenger of what went on in the intrauterine environment. The fact that we, like others before us (see Puts et al. 2008), failed to observe any associations between digit ratio and the cognitive ability outcomes further downstream in our model underscores this notion of restricted validity. Therefore, we suggest that one particularly fruitful avenue for future research is the assessment of implicit motives in individuals whose mothers have participated in amniotic-fluid studies during pregnancy (e.g., Lutchmaya et al. 2004) or who suffer from CAH, a condition that leads to elevated androgen levels during fetal development (see Puts et al. 2008). Another possibility is the investigation of changing gonadal steroid levels during puberty, another developmental phase in which, according to recent research (see Romeo 2003; Sisk and Zehr 2005), organizational effects of hormones on brain and behavior can occur and which may provide more accessible participant populations for researchers. While such studies would not yet establish a causal effect of gonadal steroids on the development of motivational need systems, they would help to link motives more directly to organizational effects of hormones. 
Another issue that needs to be addressed in future research is the examination of complex hormonal effects on motives and cognition and their potential moderation by gender. In the present study, we focused on direct associations of salivary hormones with implicit motives and cognitive abilities and controlled for gender or group status. However, current levels of gonadal steroids often interact in shaping cognition and behavior, and sometimes in a gender-specific manner, as evidenced, for instance, by animal research on joint effects of $\mathrm{T}$ and $\mathrm{E}$ in male mating behavior (e.g., Baum 2002) and research on P-attenuated effects of T and E on female aggression (e.g., Albert et al. 1992). Consistent with the notion of hormone-hormone interactions on behavior, but outside the focus of our hypotheses and therefore not reported in this paper, we observed in exploratory analyses that the interaction of $T$ and $E$ strongly predicts $n$ Power and verbal fluency in a gender-dependent manner, with very low or very high levels in both hormones predicting high verbal fluency and high levels of either hormone, but not both, predicting high $\mathrm{n}$ Power in men. Although such hormonehormone interactions are rarely studied in research with human subjects (for a notable exception, see Mehta and Josephs 2010), we suggest that it may represent a particularly fruitful approach for testing the conceptual model we introduce here in particular and associations between hormones and behavior in humans more generally.

A third issue that deserves attention in future research is the conceptually guided replication and extension of motive effects on cognitive ability measures. We think it will be particularly fruitful for future studies to examine the role of $n$ Power in spatial cognition tasks other than 3D mental rotation, the role of implicit motives in verbal fluency beyond the generation of words and simple sentences (e.g., narrative fluency, for which the implicit motive literature documents a robust gender difference favoring women; see Pang and Schultheiss 2005; Schultheiss and Brunstein 2001), and to examine in addition other cognitive abilities that are clearly sexually dimorphic (e.g., reading; see Miller and Halpern 2014). Such studies will help determine whether the role of $\mathrm{n}$ Power in mental rotation and particularly the complex implicit motive effects on verbal fluency we report here represent robust phenomena. They may also help to pinpoint those component functions inherent in such complex cognitive tasks that are specifically associated with implicit motives and activational and organizational effects of hormones.

\section{Conclusions}

To sum up, based on a conceptual framework linking organizational and activational hormone effects with implicit motives and cognitive abilities, we have examined for the first time associations between implicit motives, markers of prenatal (2D:4D digit ratio) and current (salivary T, E, and P) steroids, and cognitive abilities that have been linked to hormonal variations. We have found $\mathrm{n}$ Power to predict mental rotation performance and to be associated with digit ratio if AI, a measure of brain lateralization, is taken into account. We also found $\mathrm{n}$ Power, $\mathrm{n}$ Affiliation, and AI to jointly predict verbal fluency performance. Our findings suggest that the synthesis between the neuroendocrinology of cognitive sex differences and the endocrinology of human motivation can be used fruitfully to derive new hypotheses and insights about the role of motivational factors in cognition. 
Acknowledgments The research reported here is based in Melanie Zimni's diploma thesis. We thank Sigrid Leitmann for processing and assaying the saliva samples and Anna Rüppel for coding PSE stories.

\section{References}

Albert, D. J., Jonik, R. H., \& Walsh, M. L. (1992). Interaction of estradiol, testosterone, and progesterone in the modulation of hormone-dependent aggression in the female rat. Physiology and Behavior, 52, 773-779.

Baum, M. J. (2002). Neuroendocrinology of sexual behavior in the male. In J. B. Becker, S. M. Breedlove, D. Crews, \& M. M. McCarthy (Eds.), Behavioral endocrinology (2nd ed., pp. 153-203). Cambridge: MIT Press.

Becker, J. B., Breedlove, S. M., Crews, D., \& McCarthy, M. M. (Eds.). (2002). Behavioral endocrinology (2nd ed.). Cambridge: MIT Press.

Becker, J. B., Arnold, A. P., Berkley, K. J., Blaustein, J. D., Eckel, L. A., Hampson, E., et al. (2005). Strategies and methods for research on sex differences in brain and behavior. Endocrinology, 146, 1650-1673.

Breedlove, S. M., \& Hampson, E. (2002). Sexual differentiation of the brain and behavior. In J. B. Becker, S. M. Breedlove, D. Crews, \& M. M. McCarthy (Eds.), Behavioral endocrinology (2nd ed., pp. 75-116). Cambridge: MIT Press.

Brown, W. M., Hines, M., Fane, B. A., \& Breedlove, S. M. (2002). Masculinized finger length patterns in human males and females with congenital adrenal hyperplasia. Hormones and Behavior, 42, 380-386.

Brown, S. L., Fredrickson, B. L., Wirth, M. M., Poulin, M. J., Meier, E. A., Heaphy, E. D., et al. (2009). Social closeness increases salivary progesterone in humans. Hormones and Behavior, 56, 108-111.

Burton, L. A., Henninger, D., \& Hafetz, J. (2005). Gender differences in relations of mental rotation, verbal fluency, and SAT scores to finger length ratios as hormonal indexes. Developmental Neuropsychology, 28, 493-505.

Christensen, P. R., \& Guilford, J. P. (1958). Christensen-Guilford fluency tests (3rd ed.). Beverly Hills: Sheridan Supply Co.

Ecuyer-Dab, I., \& Robert, M. (2004). Have sex differences in spatial ability evolved from male competition for mating and female concern for survival? Cognition, 91, 221-257.

Falter, C. M., Arroyo, M., \& Davis, G. J. (2006). Testosterone: Activation or organization of spatial cognition? Biological Psychology, 73, 132-140.

Geschwind, N., \& Galaburda, A. M. (1985). Cerebral lateralization. Biological mechanisms, associations, and pathology: I. A hypothesis and a program for research. Archives of Neurology, 42, 428-459.

Gouchie, C., \& Kimura, D. (1991). The relationship between testosterone levels and cognitive ability patterns. Psychoneuroendocrinology, 16, 323-334.

Grimshaw, G. M., Sitarenios, G., \& Finegan, J. A. (1995). Mental rotation at 7 years: Relations with prenatal testosterone levels and spatial play experiences. Brain and Cognition, 29, 85-100.

Hampson, E. (1990a). Estrogen-related variations in human spatial and articulatory-motor skills. Psychoneuroendocrinology, 15, 97-111.

Hampson, E. (1990b). Variations in sex-related cognitive abilities across the menstrual cycle. Brain and Cognition, 14, 26-43.

Hampson, E. (2002). Sex differences in human brain and cognition: The influence of sex steroids in early and adult life. In J. B. Becker, S. M. Breedlove, \& D. Crews (Eds.), Behavioral endocrinology (2nd ed., pp. 579-628). Cambridge: MIT Press.

Hampson, E., Levy-Cooperman, N., \& Korman, J. M. (2014). Estradiol and mental rotation: Relation to dimensionality, difficulty, or angular disparity? Hormones and Behavior, 65, 238-248.

Hausmann, M., Slabbekoorn, D., Van Goozen, S. H., Cohen-Kettenis, P. T., \& Gunturkun, O. (2000). Sex hormones affect spatial abilities during the menstrual cycle. Behavioral Neuroscience, 114, 1245-1250.

Honekopp, J., Bartholdt, L., Beier, L., \& Liebert, A. (2007). Second to fourth digit length ratio (2D:4D) and adult sex hormone levels: New data and a meta-analytic review. Psychoneuroendocrinology, 32, 313-321.

Jemmott, J. B. (1987). Social motives and susceptibility to disease: Stalking individual differences in health risks. Journal of Personality, 55, 267-298.

Kemper, C. J., \& Schwerdtfeger, A. (2009). Comparing indirect methods of digit ratio (2D:4D) measurement. American Journal of Human Biology, 21, 188-191.

Koestner, R., \& McClelland, D. C. (1992). The affiliation motive. In C. P. Smith (Ed.), Motivation and personality: Handbook of thematic content analysis (pp. 205-210). New York: Cambridge University Press. 
Lansing, J. B., \& Heyns, R. W. (1959). Need affiliation and frequency of four types of communication. Journal of Abnormal and Social Psychology, 58, 365-372.

Liening, S. H., Stanton, S. J., Saini, E. K., \& Schultheiss, O. C. (2010). Salivary testosterone, cortisol, and progesterone: Two-week stability, interhormone correlations, and effects of time of day, menstrual cycle, and oral contraceptive use on steroid hormone levels. Physiology and Behavior, 99, 8-16.

Liu, J., Portnoy, J., \& Raine, A. (2012). Association between a marker for prenatal testosterone exposure and externalizing behavior problems in children. Developmental and Psychopathology, 24, 771-782.

Lutchmaya, S., Baron-Cohen, S., Raggatt, P., Knickmeyer, R., \& Manning, J. T. (2004). 2nd to 4th digit ratios, fetal testosterone and estradiol. Early Human Development, 77, 23-28.

Luxen, M. F., \& Buunk, B. P. (2005). Second-to-fourth digit ratio related to verbal and numerical intelligence and the big five. Personality and Individual Differences, 39, 959-966.

Mason, A., \& Blankenship, V. (1987). Power and affiliation motivation, stress, and abuse in intimate relationships. Journal of Personality and Social Psychology, 52, 203-210.

Mazur, A., \& Booth, A. (1998). Testosterone and dominance in men. Behavioral and Brain Sciences, 21, 353397.

McAdams, D. P., \& Constantian, C. A. (1983). Intimacy and affiliation motives in daily living: An experience sampling analysis. Journal of Personality and Social Psychology, 45, 851-861.

McClelland, D. C. (1987). Human motivation. New York: Cambridge University Press.

McClelland, D. C. (1989). Motivational factors in health and disease. American Psychologist, 44, 675-683.

McClelland, D. C., \& Boyatzis, R. E. (1982). Leadership motive pattern and long-term success in management. Journal of Applied Psychology, 67, 737-743.

McClelland, D. C., \& Pilon, D. A. (1983). Sources of adult motives in patterns of parent behavior in early childhood. Journal of Personality and Social Psychology, 44, 564-574.

Mehta, P. H., \& Josephs, R. A. (2010). Testosterone and cortisol jointly regulate dominance: Evidence for a dual-hormone hypothesis. Hormones and Behavior, 58, 898-906.

Miller, D. I., \& Halpern, D. F. (2014). The new science of cognitive sex differences. Trends in Cognitive Science, $18,37-45$.

Moffat, S. D., \& Hampson, E. (1996). A curvilinear relationship between testosterone and spatial cognition in humans: Possible influence of hand preference. Psychoneuroendocrinology, 21, 323-337.

Nelson, R. J. (2005). An introduction to behavioral endocrinology (3rd ed.). Sunderland: Sinauer Associates Inc.

Oxford, J., Rösch, A. G., \& Schultheiss, O. C. (in preparation). Inhibited power motivation is associated with 2D:4D digit ratio. Department of Psychology, Friedrich-Alexander University, Erlangen: Unpublished manuscript.

Pang, J. S., \& Schultheiss, O. C. (2005). Assessing implicit motives in U.S. college students: Effects of picture type and position, gender and ethnicity, and cross-cultural comparisons. Journal of Personality Assessment, 85, 280-294.

Pasterski, V., Hindmarsh, P., Geffner, M., Brook, C., Brain, C., \& Hines, M. (2007). Increased aggression and activity level in 3- to 11-year-old girls with congenital adrenal hyperplasia (CAH). Hormones and Behavior, 52, 368-374.

Patterson, F. G. P., Holts, C. L., \& Saphire, L. (1991). Cyclic changes in hormonal, physical, behavioral, and linguistic measures in a female lowland gorilla. American Journal of Primatology, 24, 181-194.

Peters, M., \& Battista, C. (2008). Applications of mental rotation figures of the Shepard and Metzler type and description of a mental rotation stimulus library. Brain and Cognition, 66, 260-264.

Phoenix, C. H., Goy, R. W., Gerall, A. A., \& Young, W. C. (1959). Organizing action of prenatally administered testosterone propionate on the tissues mediating mating behavior in the female guinea pig. Endocrinology, 65, 369-382.

Puts, D. A., McDaniel, M. A., Jordan, C. L., \& Breedlove, S. M. (2008). Spatial ability and prenatal androgens: Meta-analyses of congenital adrenal hyperplasia and digit ratio (2D:4D) studies. Archives of Sexual Behavior, 37, 100-111.

Puts, D. A., Cardenas, R. A., Bailey, D. H., Burriss, R. P., Jordan, C. L., \& Breedlove, S. M. (2010). Salivary testosterone does not predict mental rotation performance in men or women. Hormones and Behavior, 58 , 282-289.

Romeo, R. D. (2003). Puberty: A period of both organizational and activational effects of steroid hormones on neurobehavioural development. Journal of Neuroendocrinology, 15, 1185-1192.

Roof, R. L., \& Havens, M. D. (1992). Testosterone improves maze performance and induces development of a male hippocampus in females. Brain Research, 572, 310-313.

Rösch, A. G., Stanton, S. J., \& Schultheiss, O. C. (2013). Implicit motives predict affective responses to emotional expressions. Frontiers in Psychology, 4. 
Saino, N., Rubolini, D., Romano, M., \& Boncoraglio, G. (2007). Increased egg estradiol concentration feminizes digit ratios of male pheasants (Phasianus colchicus). Naturwissenschaften, 94, 207-212.

Schattmann, L., \& Sherwin, B. B. (2007). Effects of the pharmacologic manipulation of testosterone on cognitive functioning in women with polycystic ovary syndrome: A randomized, placebo-controlled treatment study. Hormones and Behavior, 51, 579-586.

Schoppe, K.-J. (1975). Verbaler kreativitäts-test [verbal creativity test]. Göttingen: Hogrefe.

Schultheiss, O. C. (2007). A biobehavioral model of implicit power motivation arousal, reward and frustration. In E. Harmon-Jones \& P. Winkielman (Eds.), Social neuroscience: Integrating biological and psychological explanations of social behavior (pp. 176-196). New York: Guilford.

Schultheiss, O. C. (2008). Implicit motives. In O. P. John, R. W. Robins, \& L. A. Pervin (Eds.), Handbook of personality: Theory and research (3rd ed., pp. 603-633). New York: Guilford.

Schultheiss, O. C. (2013). The hormonal correlates of implicit motives. Social and Personality Psychology Compass, 7, 52-65.

Schultheiss, O. C., \& Brunstein, J. C. (2001). Assessing implicit motives with a research version of the TAT: Picture profiles, gender differences, and relations to other personality measures. Journal of Personality Assessment, 77, 71-86.

Schultheiss, O. C., \& Hale, J. A. (2007). Implicit motives modulate attentional orienting to perceived facial expressions of emotion. Motivation and Emotion, 31, 13-24.

Schultheiss, O. C., \& Pang, J. S. (2007). Measuring implicit motives. In R. W. Robins, R. C. Fraley, \& R. Krueger (Eds.), Handbook of research methods in personality psychology (pp. 322-344). New York: Guilford.

Schultheiss, O. C., \& Rohde, W. (2002). Implicit power motivation predicts men's testosterone changes and implicit learning in a contest situation. Hormones and Behavior, 41, 195-202.

Schultheiss, O. C., \& Stanton, S. J. (2009). Assessment of salivary hormones. In E. Harmon-Jones \& J. S. Beer (Eds.), Methods in social neuroscience (pp. 17-44). New York: Guilford.

Schultheiss, O. C., Dargel, A., \& Rohde, W. (2003). Implicit motives and gonadal steroid hormones: Effects of menstrual cycle phase, oral contraceptive use, and relationship status. Hormones and Behavior, 43, 293301.

Schultheiss, O. C., Wirth, M. M., \& Stanton, S. J. (2004). Effects of affiliation and power motivation arousal on salivary progesterone and testosterone. Hormones and Behavior, 46, 592-599.

Schultheiss, O. C., Wirth, M. M., Torges, C. M., Pang, J. S., Villacorta, M. A., \& Welsh, K. M. (2005). Effects of implicit power motivation on men's and women's implicit learning and testosterone changes after social victory or defeat. Journal of Personality and Social Psychology, 88, 174-188.

Schultheiss, O. C., Liening, S., \& Schad, D. (2008). The reliability of a picture story exercise measure of implicit motives: Estimates of internal consistency, retest reliability, and ipsative stability. Journal of Research in Personality, 42, 1560-1571.

Schultheiss, O. C., Riebel, K., \& Jones, N. M. (2009). Activity inhibition: A predictor of lateralized brain function during stress? Neuropsychology, 23, 392-404.

Sellers, J. G., Mehl, M. R., \& Josephs, R. A. (2007). Hormones and personality: Testosterone as a marker of individual differences. Journal of Research in Personality, 41, 126-138.

Sisk, C. L., \& Zehr, J. L. (2005). Pubertal hormones organize the adolescent brain and behavior. Frontiers in Neuroendocrinology, 26, 163-174.

Smith, E. A. (2010). Communication and collective action: Language and the evolution of human cooperation. Evolution and Human Behavior, 31, 231-245.

Stanton, S., \& Edelstein, R. S. (2009). The physiology of women's power motive: Implicit power motivation is positively associated with estradiol levels in women. Journal of Research in Personality, 43, 11091113.

Stanton, S. J., \& Schultheiss, O. C. (2007). Basal and dynamic relationships between implicit power motivation and estradiol in women. Hormones and Behavior, 52, 571-580.

Stanton, S. J., \& Schultheiss, O. C. (2009). The hormonal correlates of implicit power motivation. Journal of Research in Personality, 43, 942-949.

Talarovicova, A., Krskova, L., \& Blazekova, J. (2009). Testosterone enhancement during pregnancy influences the 2D:4D ratio and open field motor activity of rat siblings in adulthood. Hormones and Behavior, $55,235-239$.

Thurstone, L., \& Thurstone, T. (1962). Primary mental abilities. Chicago: Science Research Associates.

Trivers, R., Manning, J., \& Jacobson, A. (2006). A longitudinal study of digit ratio (2D:4D) and other finger ratios in Jamaican children. Hormones and Behavior, 49, 150-156.

Vandenberg, S. G., \& Kuse, A. R. (1978). Mental rotations, a group test of three-dimensional spatial visualization. Perceptual and Motor Skills, 47, 599-604. 
Winter, D. G. (1973). The power motive. New York: Free Press.

Winter, D. G. (1994). Manual for scoring motive imagery in running text (4 ed.). Department of Psychology, University of Michigan, Ann Arbor: Unpublished manuscript.

Wirth, M. M., \& Schultheiss, O. C. (2006). Effects of affiliation arousal (hope of closeness) and affiliation stress (fear of rejection) on progesterone and cortisol. Hormones and Behavior, 50, 786-795.

Wirth, M. M., Welsh, K. M., \& Schultheiss, O. C. (2006). Salivary cortisol changes in humans after winning or losing a dominance contest depend on implicit power motivation. Hormones and Behavior, 49, 346-352.

Wolf, O. T., \& Kirschbaum, C. (2002). Endogenous estradiol and testosterone levels are associated with cognitive performance in older women and men. Hormones and Behavior, 41, 259-266. 